\title{
Un samedi du bout des lèvres
}

C.-F. Robert

Un bricoleur du samedi matin: il manipulait une cartouche de gaz, l'a laissé se vider, se la coince entre les dents pour prendre un outil. Le froid la colle aux lèvres avant qu'il ne l'arrache en hurlant. Une cloque, douleurs et l'air penaud en me racontant ses malheurs. Un gel protecteur, un anti-inflammatoire et la tournée reprend.

C'est novembre sur la ville et je circule entre les otites, les toux et les sinusites.

C'est marrant, j'ai le feeling avec les boîtes aux lettres. Rien qu'à leur allure, un nom étranger griffonné au crayon ou les huit patronymes différents dans le même trois pièces, j'arrive à prédire le problème: le gosse enrhumé et surinfecté dans la fumée des cigarettes, le travailleur de force au dos bloqué, la vendeuse qui a mal partout dans le ventre. Pourquoi faut-il qu'ils habitent toujours au sixième étage sans ascenseur?

Immeuble tranquille, escaliers en bois, une rampe patinée à laquelle je me hisse d'une main, l'autre à la traîne de ma valise de garde.

J'aime bien les petites vieilles. Elles alignent leurs boîtes à médicaments avec ordre sur l'armoire de la cuisine. Elle a appelé la police, mais ne se rappelle plus pourquoi. Je passe en revue les meubles du salon. Entre les plantes vertes, le mari est là, en noir et blanc, dans un cadre en bois.

- Votre mari vous a quitté depuis longtemps? Une attaque?

Elle se raccroche à sa vie. Il a travaillé à la poste, pendant 40 ans. C'était dur, la crise, puis la mobilisation. Les enfants sont à Olten.

- Il me reste encore de l'Adelphan-Esidrex que lui avait prescrit le Dr Wildi. Vous ne l'avez pas connu? Lui, il faisait tout: accouchements, chirurgie, médecine générale. Pas comme maintenant, avec tous ses spécialistes, on ne sait plus quoi leur dire ...

On cause un moment: je prie pour que le «beeper» ne sonne pas, juste pour préserver le calme, l'instant qui nous lie au passé et explique peutêtre le présent. J'ai même droit au thé et aux bricelets. Je régresse: je suis chez ma grand-mère et un jour je serai médecin. Je me ressaisis.

- Vous permettez?

Correspondance: Dr Claude-François Robert Case postale 1111 CH-1211 Genève 26

Je glisse le stéthoscope sous cet attirail compliqué de corsets et de soutien-gorge usé par les lavages. Petit budget, AVS, que mange-t-elle, des biscuits, une tartine le matin?
- Merci, Docteur, cela m'a fait beaucoup de bien de vous voir.

Elle me serre la paume avec ses deux mains, comme si elle n'avait pas vu un être humain depuis longtemps. Elle ne savait plus ce qui la faisait souffrir. Il ne lui restait que l'oubli pour passer le temps.

Beaux quartiers, belle villa, tristesse infinie.

- Vous comprenez Docteur, ma femme voulait voir le médecin. Enfin, j'ai pensé que ce serait mieux pour elle, à son air ce matin. Je lui ai dit, nous sommes samedi, alors trouver un Docteur... Je vous avertis tout de suite, elle est suivie par le Professeur Koch de Bâle. Je n'ai pas pu l'atteindre, ni le Professeur Martin qui le remplace quand il est en congrès. Alors, j'ai pensé, pourquoi pas un médecin de garde?

Il parle. Il a une entreprise qui exporte jusqu'en Asie et une femme avec une sorte de vague à l'âme inexplicable.

Elle est assise dans un fauteuil, les yeux dans le vide, mélancolique à souhaits. Elle tripote un fil de laine, avec deux doigts, le faisant tourner, sans jamais bouger une ride. Elle a ce regard bleu des puits sans fonds.

- Vous savez, nous avons consulté les meilleurs spécialistes ...

J'imagine qu'ils se sont perdus, eux aussi, dans les deux faces de ce couple.

Il me dresse la liste des traitements, des molécules - il a une formation de chimiste -, les cures, les bains, les séjours à la montagne.

Je me suis approché d'elle, mais il devient plus agité. Alors, je l'expédie me préparer un café à la cuisine.

Je suis tout près; elle refuse mon regard. Je tente une phrase:

- Je vois que vous nous avez écouté, mais que vous n'avez rien dit...

Elle esquisse un sourire, juste une tension dans la commissure des lèvres.

- Vous savez, mon mari parle, et pendant tout ce temps, je continue de souffrir.
Des lèvres collées par le froid et maintenant des lèvres serrées sur le silence, qui susurrent juste quelques mots. Qu'ai-je gagné aujourd'hui? 